\title{
MiR-34a promotes fibrosis of hepatic stellate cells via the TGF- $\beta$ pathway
}

\author{
Jie Zhang ${ }^{1,2}$, Haixia Wang ${ }^{3}$, Linlin Yao ${ }^{4,5}$, Peng Zhao ${ }^{4,5 \#}$, Xiaoyan $\mathrm{Wu}^{4,5 \#}$ \\ ${ }^{1}$ Department of Nutrition, Shandong Provincial Hospital, Cheeloo College of Medicine, Shandong University, Jinan, China; ${ }^{2}$ Department of \\ Nutrition, Shandong Provincial Hospital Affiliated to Shandong First Medical University, Jinan, China; ${ }^{3}$ Healthcare Department, The Third \\ Hospital of Jinan, Jinan, China; ${ }^{4}$ Department of Cardiology, Shandong Provincial Hospital, Cheeloo College of Medicine, Shandong University, \\ Jinan, China; ${ }^{5}$ Department of Cardiology, Shandong Provincial Hospital Affiliated to Shandong First Medical University, Jinan, China \\ Contributions: (I) Conception and design: J Zhang, H Wang; (II) Administrative support: P Zhao, X Wu; (III) Provision of study materials or patients: \\ J Zhang, L Yao; (IV) Collection and assembly of data: J Zhang, H Wang, P Zhao, X Wu; (V) Data analysis and interpretation: P Zhao, X Wu; (VI) \\ Manuscript writing: All authors; (VII) Final approval of manuscript: All authors. \\ \#These authors contributed equally to this work. \\ Correspondence to: Peng Zhao, MD, PhD; Xiaoyan Wu, MD, PhD. Department of Cardiology, Shandong Provincial Hospital, Cheeloo College of \\ Medicine, Shandong University, Jinan 250021, China. Email: pengalfie@163.com; wxy_all@126.com.
}

Background: Previous studies have confirmed that MicroRNA (miRNA) is a key regulator exhibiting different effects in human liver fibrosis. However, the function of miR-34a in liver fibrosis has not been reported. Hence, this study aimed to investigate the regulatory mechanism of miR-34a in liver fibrosis.

Methods: The expression of miR-34a was measured in fibrosis tissues via the quantitative real-time PCR (qRT-PCR) assay. Subsequently, 30 male C57BL/6J mice were divided into control and treatment groups and used to establish animal models of liver fibrosis to explore the expression level of miR-34a. Moreover, Cell Counting Kit 8 (CCK-8) and transwell assays were preformed to identify the regulatory mechanism of miR-34a in cells. The effect of miR-34a on the activity of transforming growth factor- $\beta$ (TGF- $\beta$ ) pathway was observed by western blot.

Results: Up-regulation of miR-34a was detected in fibrosis cells. Moreover, the cellular phenotype was suppressed by miR-34a down-regulation in a primary culture of hepatic stellate cells (HSCs). Besides, it was found that increased miR-34a could significantly promote the invasion and migration of HSCs. Moreover, miR-34a activates HSCs through transforming TGF- $\beta$, $\alpha$-smooth muscle actin ( $\alpha$-SMA), and Monocyte chemoattractant protein-1 (MCP-1), which further affects liver fibrosis.

Conclusions: MiR-34a promotes the fibrosis of HSCs as well as cell proliferation, migration, and invasion.

Keywords: MiR-34a; liver fibrosis; Bifico; transforming growth factor- $\beta$ (TGF- $\beta$ ); hepatic stellate cells (HSCs)

Submitted Jul 07, 2021. Accepted for publication Oct 19, 2021.

doi: 10.21037/atm-21-5005

View this article at: https://dx.doi.org/10.21037/atm-21-5005

\section{Introduction}

The histopathological manifestations of cirrhosis are extensive necrosis of hepatocytes, nodular regeneration of residual hepatocytes, hyperplasia of connective tissue, and formation of fibrous septa, resulting in serious damage to the hepatic lobule structure and the aberrant generation of pseudolobule (1-3). There are approximately 3 million or more patients who suffer chronic liver disease worldwide, with a huge unmet need for treatment (4). Liver fibrosis can be defined as the progressive accumulation of extracellular matrix (ECM) and the reduction of its remodeling, which leads to the eventual destruction of the normal structure of the liver (5). Liver fibrosis is an early reversible stage of cirrhosis. If left untreated, it can develop into cirrhosis, leading to organ failure and death (6).

Liver fibrosis is a dynamic process involving the synthesis 
of matrix components and the reduction of physiological matrix decomposition (7). During the formation of liver fibrosis, hepatic stellate cell (HSC) activation is an important molecular event and a determinant of the occurrence of liver fibrosis (8-10). The HSC activation process is highly complex, and is the synergistic effect of various cytokines secreted by Kupffer cells, stem cells, hepatic sinus endothelial cells, and HSC paracrine nuclei (11). In the initial stage of HSC activation, cells undergo early changes in gene expression and phenotype, and some soluble stimuli (such as oxidative stress signals, apoptotic bodies, and lipopolysaccharides) are involved. In the subsequent stages, some specific phenotypic changes occur in HSCs, including proliferation, contractile, chemotaxis, fibrogenesis, matrix degradation, loss of retinoids, as well as the release of leukocyte chemoattractant proteins and cytokines, which directly or indirectly maintain cell activation and lead to continuous fibrosis $(5,11,12)$. Among them, transforming growth factor (TGF- $\beta$ ) is an important cytokine promoting liver fibrosis, which mainly enhances the aberrant activation of HSCs through the Smad protein signaling pathway. Specific blocking of Smad3 protein expression may inhibit liver fibrosis (13). MicroRNA consists of 21-25 nucleotides (14), and is involved in the shearing of message RNA (mRNA), inhibiting or enhancing the expression of its regulatory genes in the translation process, so as to participate in the processes of ontogeny, body metabolism, as well as the occurrence and development of diseases (15-17).

MicroRNA plays a pivotal role in maintaining the normal physiological functions of organ systems. Defects and mutations in the target sites of the maturation process of microRNA or the mRNA of its target genes can lead to abnormal functions and diseases. Numerous microRNAs (miRNAs) are expressed in HSCs and control the progression of fibrosis, including miR-29a (18), miR-19b (19), and miR-221/222 (20). Based on gene array analysis, miR-29a, an inhibitor of a variety of extracellular matrix (ECM) proteins, can be down-regulated by TGF- $\beta$ and LPS (18). miR-19b has been found to inhibit the TGF- $\beta$ pathway, which is reduced in the tissues of patients with advanced fibrosis, while it is overexpressed in blocking HSC activation (19). In contrast, the up-regulated expression of miR-221/222 in the human liver is parallel to the progression of liver fibrosis (20). Its expression also increases with the activation of HSCs, and its participation in HSC proliferation has been confirmed by researchers. MiR-34a has been found to play key roles in the fibrosis of multiple cells. Cui et al. found that miR-34a could promote fibrosis in aged lungs via blocking the functions of alveolar epithelial cells (21). Bifico is used to treat diarrhea and abdominal distention caused by the imbalance of intestinal flora, and is also applied in the treatment of mild to moderate acute diarrhea. Bifico is made up of Bifidobacteria, Lactobacillus acidophilus, and Enterococcus faecalis. Some researchers have demonstrated that Bifico can improve gastritis caused by pyloric spiral sensation (22). Previous study reported that the use of Bifico could reduce the plasma ammonia level in patients with cirrhosis and be used as an adjuvant therapy for cirrhosis. However, whether Bifico is involved in inhibiting cirrhosis and the formation of liver fibrosis is currently unknown.

Based on previous studies, we selected miR-34a as the target and verified the effect of miR-34a on the fibrosis of HSCs through gene expression regulation, CCK-8, and other experiments. We present the following article in accordance with the ARRIVE reporting checklist (available at https://dx.doi.org/10.21037/atm-21-5005).

\section{Methods}

\section{Sample preparation}

The samples of the 13 healthy donors and 20 patients with liver fibrosis from Shandong Provincial Hospital Affiliated to Shandong First Medical University were used in our experiments. Written consent was obtained prior to sample collection, and the Ethics Committee of the Shandong Provincial Hospital Affiliated to Shandong First Medical University approved this study. The experiment followed the Declaration of Helsinki (as revised in 2013) (23). Tissue biopsies were immediately frozen at $-80^{\circ} \mathrm{C}$.

\section{Animal models of liver fibrosis}

Animal experiments were approved by the Animal Experimentation Ethics Committee of Shandong Provincial Hospital Affiliated to Shandong First Medical University. Moreover, all experiments in this study were performed in strict accordance with the Animal Experimentation Ethics Committee Guide for the Care and Use of Laboratory Animals. A protocol was prepared before the study without registration. 30 male C57BL/6J mice (Chengdu Dashuo, China), aged 8 to 10 weeks and weighing $20-25 \mathrm{~g}$, were randomly divided as control and treatment groups, and then the mice in treatment group were injected intraperitoneally 
with a solution of $\mathrm{CCl}_{4}$ (1:1 in olive oil) twice a week $(0.2 \mathrm{~mL} / 100 \mathrm{~g})$, while mice only treated with olive oil were used as controls. After intervention for 8 weeks, the mice were anaesthetized and sacrificed, and the liver tissues were obtained and frozen for subsequent experiments.

\section{Cell culture}

All cell lines including LX-1 and HSC-T6 were purchased from Tongpai (Shanghai) Biotechnology Co., Ltd (Shanghai, China), and all cells exhibited the normal functions and phenotype. For rat HSCs (rHSCs) preparation, the liver tissues of male Sprague-Dawley rats were obtained and then digested with collagenase/pronase, and the cells were then centrifugated and isolated by continuous density gradients. Next, the HSCs were cultured on plastic culture dishes with Dulbecco's modified Eagle's medium [DMEM; GIBCO, Grand Island, NY; 10\% fetal bovine serum (FBS)], and the cells were spontaneously activated after culturing for 7 days.

\section{Cell transfection}

MiR-34a mimic, miR-34a silence vectors and the related negative control (Shanghai Yubo Biotechnology Co., Ltd., Shanghai, China) were used for HSC transfection (LX-1, HSC-T6), and Lipofectamine 2000 (Invitrogen, Carlsbad, CA) was used as a transfection reagent. Also, the transfection efficiency of miR-34a was measured with qRTPCR after cell culture for 48 and 72 hours, respectively.

\section{Transwell migration assay}

Cellular migration was observed using a transwell assay. Briefly, $3 \times 10^{4}$ cells/well HSCs were cultured with serum-free medium in the upper chambers. The medium containing $10 \% \mathrm{FBS}$ was added into the lower chambers. The cells were cultured at $37^{\circ} \mathrm{C}$ and $5 \% \mathrm{CO}_{2}$. After culturing for 24 hours, the cells on the lower surface of upper chambers were fixed and then strained with $0.1 \%$ crystal violet, and the cells were imaged and counted under a light microscope (OPTIKA, Thermo Fisher Scientific, USA).

\section{Quantitative real-time PCR}

Total RNAs of the cells or tissues were collected using QIAzol reagent (Qiagen, Hilden, Germany). qRT-PCR was performed with SYBR Green master mixture on HT7900 system (Applied Biosystems, Foster City, CA,
USA), and U6 was used as an internal control. Moreover, the comparative $\Delta \mathrm{Ct}$ method was performed to evaluate the expression of miR-34a.

\section{Western blot}

Total proteins of all cells or tissues were extracted using RIPA Lysis buffer, and the concentrations of extracts were then measured using Bicinchoninic acid (BCA) kits (Shanghai Jining Biotechnology Co., Ltd., Shanghai, China). The samples of $30 \mu \mathrm{g}$ boiled proteins proteins were added and separated with $12 \%$ sodium dodecyl sulfatepolyacrylamide gel electrophoresis (SDS-PAGE), and the proteins were then transferred onto the nitrocellulose membranes (GE Healthcare, Piscataway, NJ, USA) using wet transfer methods.

Subsequently, the membranes were blocked with $5 \%$ fat-free milk for 2 hours, and then all membranes were incubated with the related primary antibodies at $4{ }^{\circ} \mathrm{C}$ overnight. Finally, the membranes were incubated with the second antibodies, and the expressions of the proteins were measured under a chemiluminescence imaging system (Invitrogen iBright, Thermo Fisher Scientific, USA).

\section{Cell viability assay}

$3 \times 10^{3}$ of the cells were cultured into 96-well plates, and the [3-(4,5-dimethylthiazol2-yl)-5-(3-carboxymethoxyphenyl)2-(4-sulfophenyl)-2Htetrazolium, inner salt] (MTS) assay was used to observed the viability of the cells. Briefly, the cells in each well were incubated with $20 \mu \mathrm{L}$ of reaction solution at $37^{\circ} \mathrm{C}$ for 1.5 hours. Finally, the absorbance of the cells was measured at $490 \mathrm{~nm}$.

\section{Statistical analysis}

All experiments in this study were independently performed three times. The data were analyzed using the Student's t-test for single, and were presented as mean \pm standard deviation. $\mathrm{P}<0.05$ was considered to be statistically significant.

\section{Results}

\section{The expression of miR-34a in hepatic fibrosis tissues}

Firstly, to explore the role of miR-34a in hepatic fibrosis tissues, the relative levels of miR-34a in the fibrosis and 


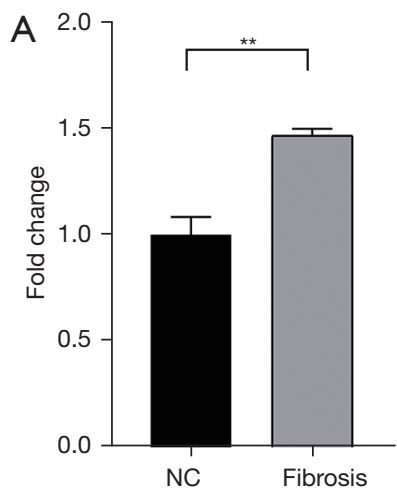

B
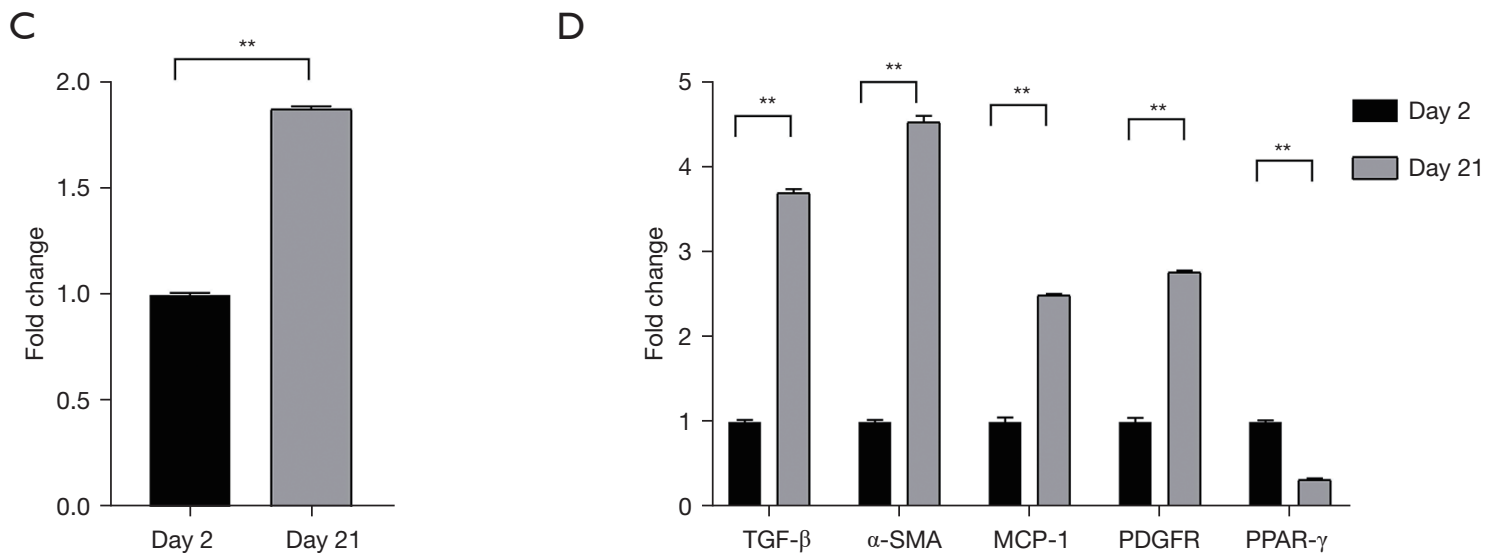

Figure 1 MiR-34a expression was increased in liver fibrosis tissue. (A) The level of miR-34a in liver tissue; (B) the fibrosis morphology of HSCs cultured in vitro changed over time; (C) miR-34a was up-regulated in the in vitro cultured HSCs at 21 days; (D) TGF- $\beta$, $\alpha$-SMA, MCP-1, and PDGFR were up-regulated on day 21. Scale bar $=100 \mu \mathrm{m} .{ }^{* *}, \mathrm{P}<0.01$. HSCs, hepatic stellate cells.

normal tissues were measured by qPCR. Compared with the tissues of healthy donors, the level of miR-34a in fibrosis tissues was obviously higher (Figure 1A). HSCs cells in the primary culture developed fibrosis and cell morphology changes at 21 days (Figure 1B). qPCR detection of primary cultured HSCs on day 2 and day 21 showed that the miR34a expression increased after HSC fibrosis (Figure 1C). MRNA expression was measured for genes involved in HSC activation, such as $\alpha$-SMA, TGF- $\beta$, platelet-derived growth factor receptor (PDGFR), peroxisome proliferatoractivated receptor (PPAR- $\gamma$ ), and monocyte chemoattractant protein-1 (MCP-1), which were increased at 21 days. This demonstrated that the primary cultured HSCs developed fibrosis at 21 days (Figure 1D).

\section{Overexpression of miR-34a accelerated HSCs fibrosis in vitro}

In the in vitro cultured HSCs, miR-34a with strong promoter
CMV was transfected to overexpress miR-34a (Figure $2 A$ ). Compared with the cells transfected with negative control (NC) of miR-34a, the expression level of the HSC activation marker in cells that overexpressed miR-34a was increased (Figure 2B). The cell morphology was fibrosis on the $7^{\text {th }}$ day after transfection, and notable fibrosis was found on the $14^{\text {th }}$ day. Meanwhile, obvious fibrosis was not found in the control group (Figure 2C).

\section{Decreased miR-34a inbibited HSCs fibrosis}

MiR-34a was down-regulated in HSCs by RNA interference technology targeting the miR-34a sequence (Figure $3 A$ ). Adding miR-34a RNA interference to cultured HSCs could inhibit HSC fibrosis in vitro (Figure 3B). Western blot experiments demonstrated that the decreased miR34a could impede the translation of gene-related proteins activated by HSCs (Figure 3C,3D). 


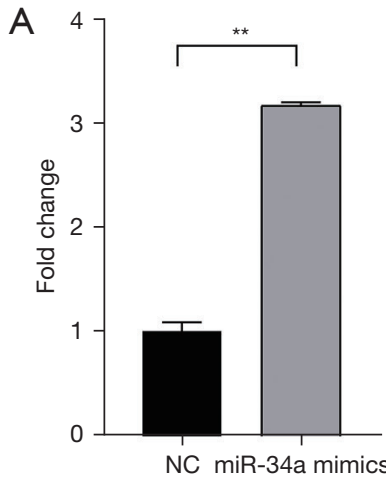

C

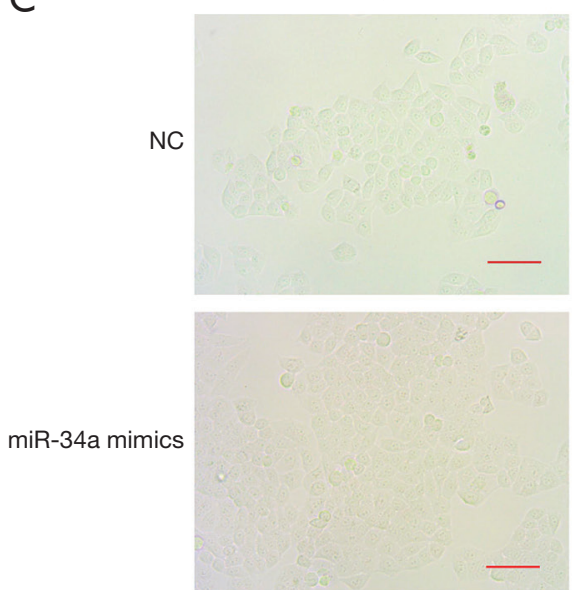

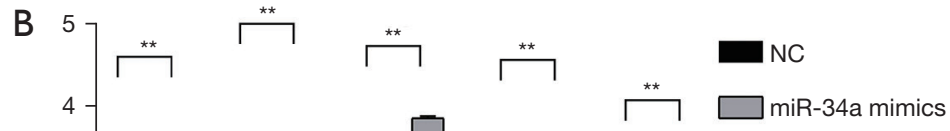

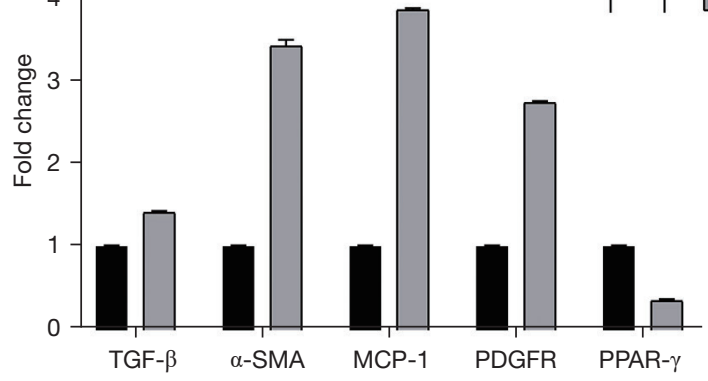

Day 7

Day 14
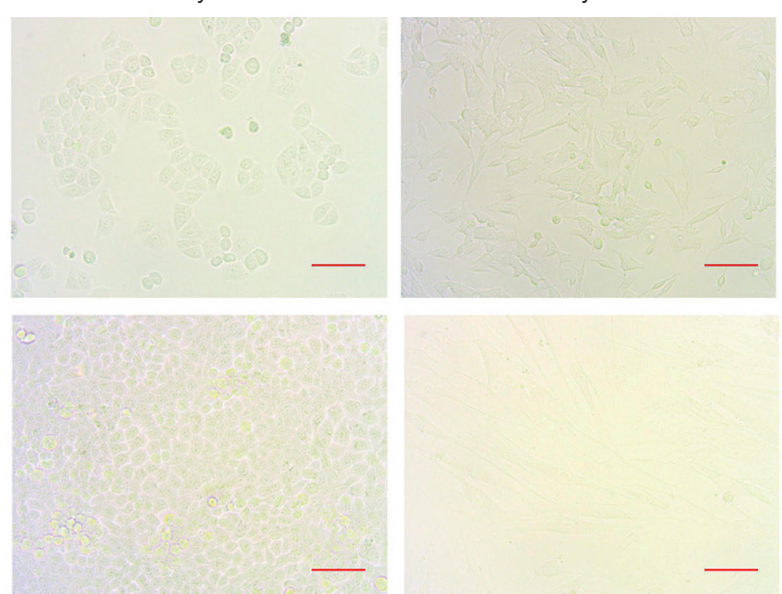

Figure 2 Overexpression of miR-34a promotes fibrosis of HSCs. (A) The level of miR-34a in the HSCs transfected with miR-34a mimics; (B) TGF- $\beta$, $\alpha$-SMA, MCP-1, and PDGFR were up-regulated by miR-34a mimics; (C) HSCs that overexpressed miR-34a became fibrotic at 14 days. Scale bar $=100 \mu \mathrm{m}$. ${ }^{* *}, \mathrm{P}<0.01$. HSCs, hepatic stellate cells.

\section{Overexpression of miR-34a promoted the proliferation, migration and invasion of HSCs}

To further investigate the role of miR-34a in the proliferation, migration of HSCs, CCK- 8 and transwell assay were used to detect cell proliferation and migration ability. Using the CCK-8 assay, we found that downregulation of miR-34a significantly suppressed cellular proliferation, while overexpression of miR-34a increased proliferation in HSCs (Figure 4A). Using the transwell assay, up-regulation of miR-34a notably increased the invasion and migration in HSCs (Figure 4B,4C).

\section{Knockdown of miR-34a inbibited $\mathrm{CCl}_{4}$-induced liver fibrosis}

The expression of miR-34a was significantly increased in the isolated liver tissues of the mice after treatment with $\mathrm{CCl}_{4}$ (Figure $5 \mathrm{~A}, 5 \mathrm{~B}$ ). We also found that the injection of miR-34a siRNA in the mice treated with $\mathrm{CCl}_{4}$ could knockdown the expression level of miR34a (Figure 5C,5D). Similarly, $\mathrm{CCl}_{4}$ induction increased the expression of HSC activated proteins, while miR34a siRNA injection decreased the expression of proteins (Figure 5C,5D).

\section{Treatment with Bifico can inbibit liver fibrosis}

Gavage of Bifico to $\mathrm{CCl}_{4}$-induced mice decreased the expression of miR-34a in the liver (Figure 6A). Through western blot detection, we observed that Bifico could inhibit the expression of the activated proteins and further inhibit the fibrosis of HSCs (Figure 6B,6C). 
A

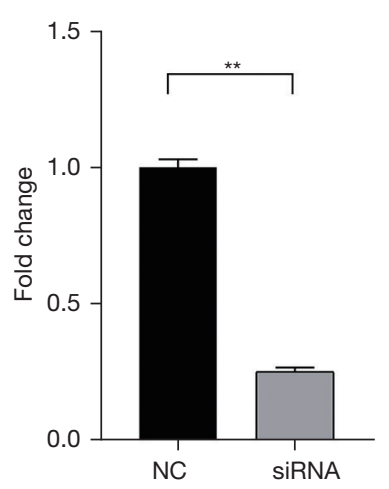

B

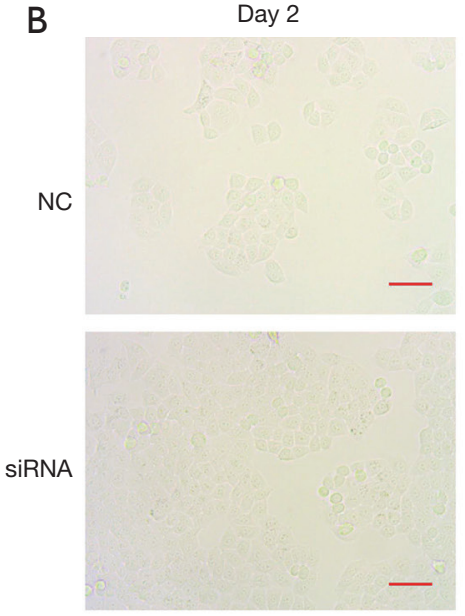

C

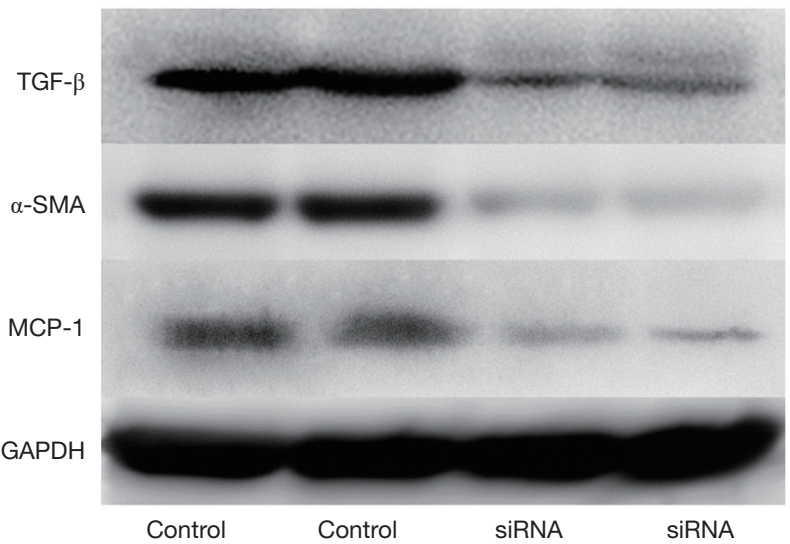

Day 7
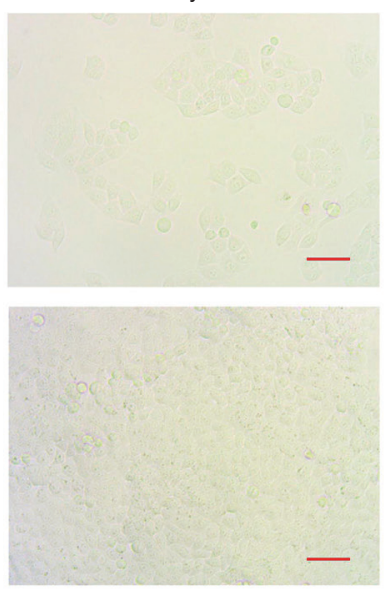

D

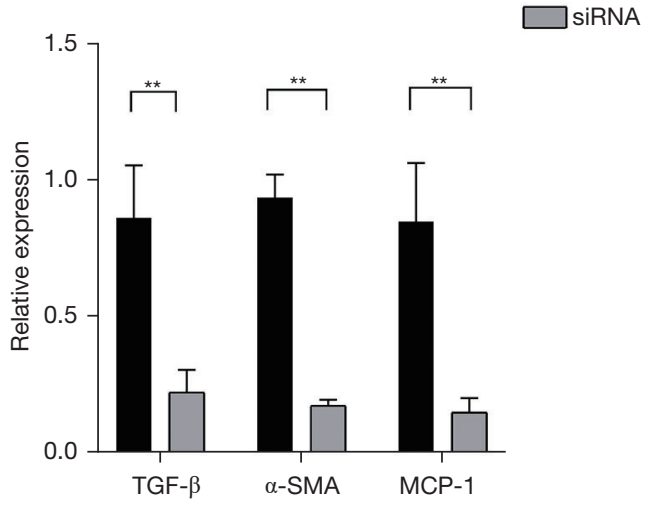

Figure 3 Knockdown of miR-34a impeded the fibrosis of HSCs. (A) The level of miR-34a in the HSCs transfected with miR-34a siRNA; (B) the morphology of HSCs with low miR-34a expression did not change with fibrosis at 14 days; (C) TGF- $\beta, \alpha-S M A$, and MCP-1 were downregulated on day 14 in miR-34a knockdown HSCs; (D) the relative expression levels of TGF- $\beta, \alpha-S M A$, and MCP-1 decreased in miR-34a knockdown HSCs. Scale bar $=100 \mu \mathrm{m} .{ }^{* *}, \mathrm{P}<0.01$.

\section{Discussion}

HSCs play a role in the formation of the matrix of cells, the regulation of hepatic sinus blood flow and hepatocyte regeneration, and are also related to the absorption and utilization of vitamin A. At present, the phenotypic activation of HSCs is considered to be a key pathological process in the formation of liver fibrosis (24). Lipid droplets in the cytoplasm decrease or disappear. HSCs are converted to myofibroblast cells (MFB), which expresses smooth muscle $\alpha$-actin ( $\alpha$-SMA) and leads to the increase of the $\mathrm{Na}^{+} / \mathrm{H}^{+}$exchange level. The number of activated HSCs is a central condition for liver fibrosis. HSCs is produced in the process of liver fibrosis, and extracellular matrix (ECM) secreted from HSCs promote the formation of liver fibrosis and eventually lead to cirrhosis of the liver, which is the main cause of liver failure. Therefore, there are two strategies for inhibiting the fibrosis of liver including inhibiting the activation of HSCs, and promoting HSC apoptosis $(5,7,10,25)$.

The TGF- $\beta /$ Smads pathway is the most classic and common pathway for the mechanism of action of TGF- $\beta$ (26). Smads protein is the central link of the TGF- $\beta$ signal transduction pathway, which is a TGF- $\beta$ receptor, and is involved in the regulation of cellular proliferation, 

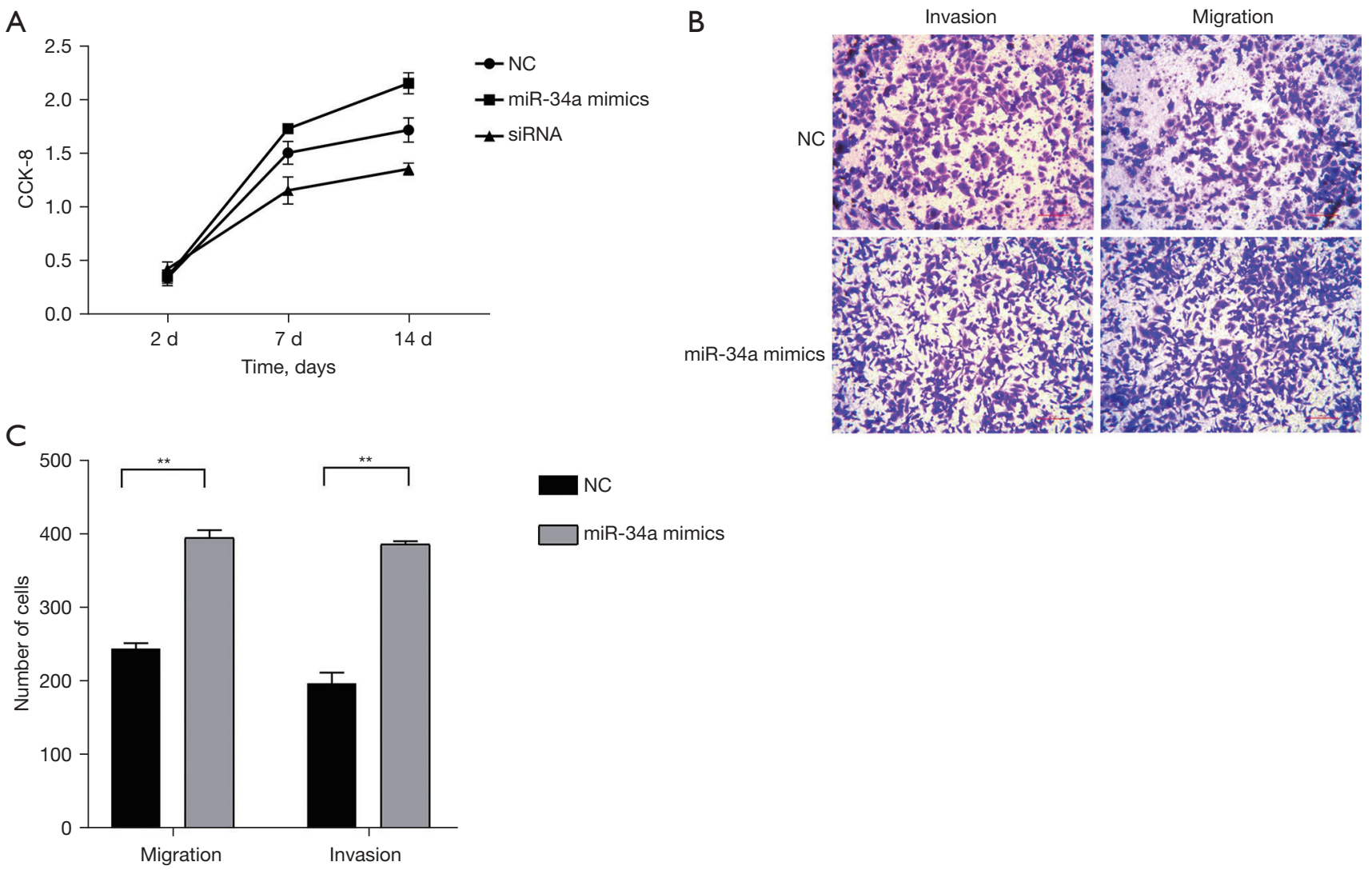

Figure 4 Overexpression of miR-34a promoted HSCs proliferation, migration and invasion. (A) The proliferation of HSCs with decreased miR-34a was significantly inhibited; $(B, C)$ the representative images and quantification of the effects of increased miR-34a on cellular invasion and migration in HSCs. Scale bar $=100 \mu \mathrm{m}$; Staining: $1 \%$ crystal violet. ${ }^{* *}, \mathrm{P}<0.01$. HSCs, hepatic stellate cells.

transformation, synthesis, secretion, and apoptosis $(13,27)$.

Numerous miRNA molecules have been found to regulate the TGF- $\beta$ pathway (28). For instance, miR-33a has a pro-fibrotic effect and TGF- $\beta$ can up-regulate the level of miR-33a via the PI3K/AKT signaling pathway (29). Our study demonstrated a notable increase in miR-34a level in hepatic fibrosis tissues. Considering that the upregulation of miR-34a exists in many liver diseases and that TGF- $\beta$ is frequently related, we hypothesize that the activation of miR-34a/TGF- $\beta$ pathway is related to HSCs and plays a role in the progress of liver fibrosis. Data also showed that overexpression of miR-34a promoted fibrosis of HSCs while down-regulation of miR-34a inhibited fibrosis of HSCs. Moreover, cell proliferation, migration and invasion were suppressed by miR-34a downregulation in primary culture of HSCs. TGF- $\beta$ can also promote the expression of miR31 through the Smad3 signaling pathway, and miR-31 is also a pro-fibrosis factor, which can activate HSCs by down- regulating hypoxia-inducing factor 1 inhibiting molecule (FIH1) (30). TGF- $\beta$ can promote the occurrence of liver fibrosis by up-regulating miR-199a to inhibit the expression of liver cell growth factor (HGF). On the other hand, TGF- $\beta$ can down-regulate the expression of some antifibrotic miRNAs (such as miR-150) in HSCs, as well as the expression of miR-150 in HSCs. The anti-fibrotic effect of miR-150 is related to its ability to directly bind to collagen type I and type IV genes. We wondered to know whether the crosstalk between miR-34a and TGF- $\beta$ exists. We found that miR-34a activated HSCs through TGF- $\beta, \alpha$-SMA and MCP-1, further affecting liver fibrosis. Our data indicated that the miR-34a/TGF- $\beta$ signaling pathway participated in the process of liver fibrosis. This signaling pathway may be a potential treatment target for liver fibrosis.

The dysfunction of probiotics has been confirmed as a major cause of multiple diseases. In this study, Bifico was fed to the mice, and we observed that it could decrease 
A
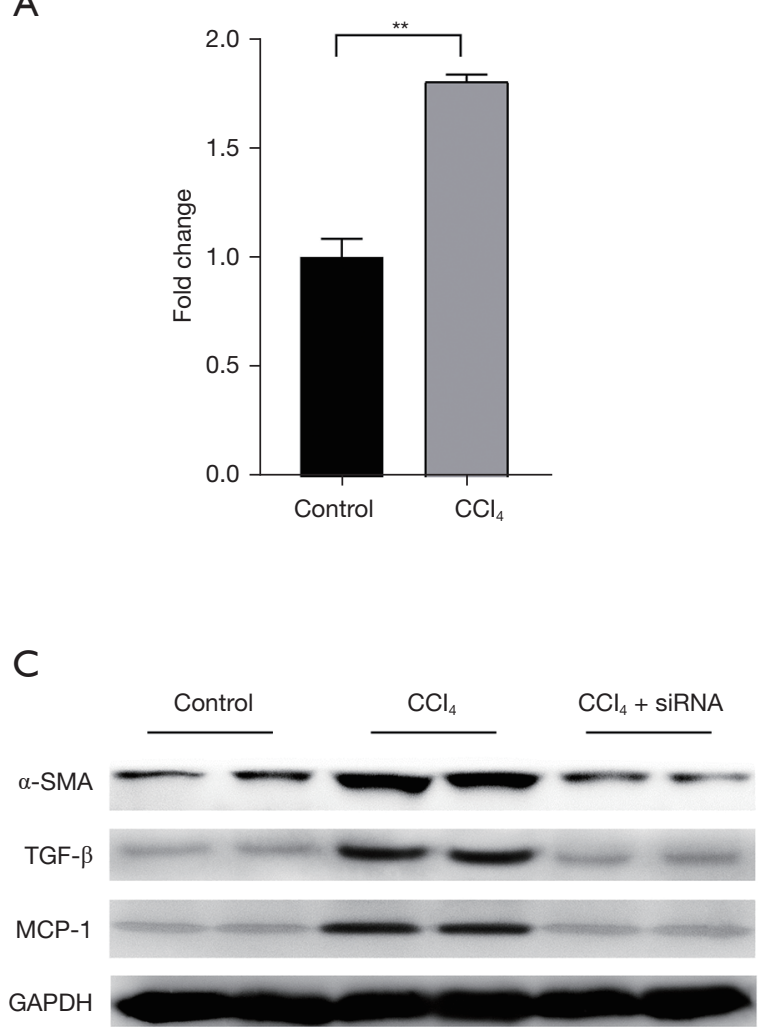

B

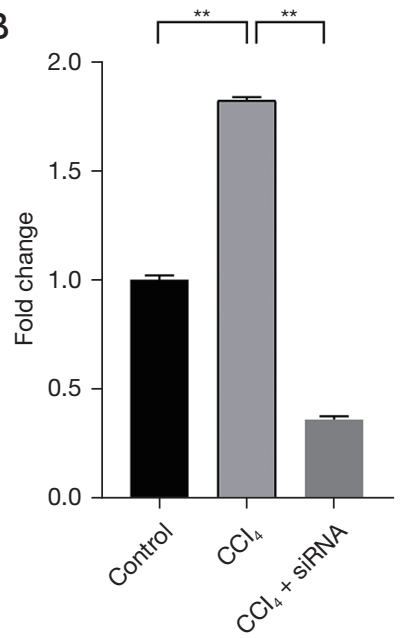

D

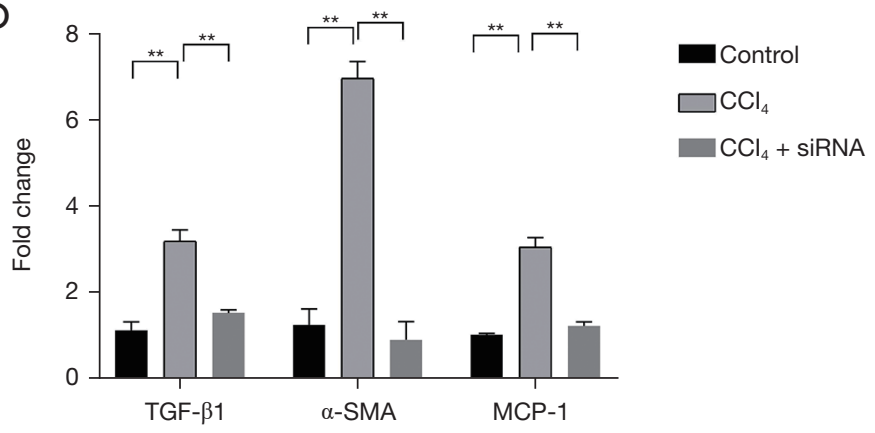

Figure 5 Knockdown of miR-34a inhibited $\mathrm{CCl}_{4}$-induced liver fibrosis. (A) $\mathrm{CCl}_{4}$-induced increased miR-34a expression in mice; (B) the addition of miR-34a siRNA inhibited the increase of miR-34a expression in $\mathrm{CCl}_{4}$-induced mice; (C,D) $\mathrm{CCl}_{4}$-induced increased TGF- $\beta$, $\alpha$-SMA, and MCP-1 in mice. After injecting with $\mathrm{CCl}_{4}$ and siRNA, the expression of TGF- $\beta, \alpha$-SMA, and MCP- 1 was decreased. ${ }^{* *}, \mathrm{P}<0.01$.

the level of miR-34a and inhibit the progression of liver fibrosis. Bifico is composed of Bifidobacteria, Lactobacillus acidophilus, and Enterococcus faecalis. Previous studies have indicated that deficiency of these probiotics is closely related to liver dysfunction, including liver inflammation and cirrhosis $(31,32)$. It seems that the aberrant level of
miR-34a is related to probiotic imbalance, although more evidence is necessary to validate this hypothesis.

In conclusion, this study revealed the mechanism of liver fibrosis development to some degree, and suggested that miR-34a could activate the TGF- $\beta$ signaling pathway and the fibrosis of HSCs. This provides a target for the 
A

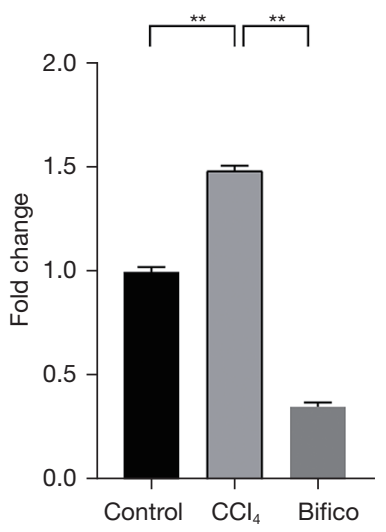

B

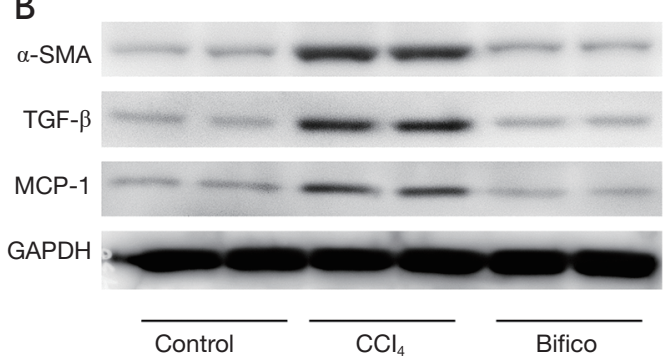

C

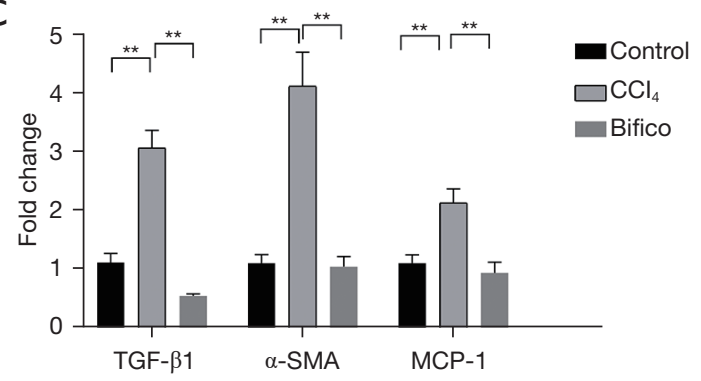

Figure 6 Treatment with Bifico could inhibit liver fibrosis. (A) The addition of Bifico inhibited the increase of miR-34a expression in $\mathrm{CCl}_{4^{-}}$ induced mice; (B,C) Bifico inhibited the expression of HSC activation marker proteins TGF- $\beta, \alpha-S M A$, and MCP-1. **, $\mathrm{P}<0.01$.

treatment and prevention of liver fibrosis.

\section{Acknowledgments}

Funding: Provincial Natural Science Foundation of Shandong province, China (ZR2020QH019) to JZ; Traditional Chinese Medicine Science and Technology Project of Shandong Province (2020M041), Clinical Medicine Science and Technology Innovation Plan of Jinan City (202019093), Medical Health Science and Technology Development Plan of Shandong Province (2019WS496) to PZ.

\section{Footnote}

Reporting Checklist: The authors have completed the ARRIVE reporting checklist. Available at https://dx.doi. org/10.21037/atm-21-5005

Data Sharing Statement: Available at https://dx.doi. org/10.21037/atm-21-5005

Conflicts of Interest: All authors have completed the ICMJE uniform disclosure form (available at https://dx.doi. org/10.21037/atm-21-5005). The authors have no conflicts of interest to declare.

Ethical Statement: The authors are accountable for all aspects of the work in ensuring that questions related to the accuracy or integrity of any part of the work are appropriately investigated and resolved. Animal experiments were approved by the Animal Experimentation Ethics Committee of Shandong Provincial Hospital Affiliated to Shandong First Medical University. All experiments in this study were performed in strict accordance with the Animal Experimentation Ethics Committee Guide for the Care and Use of Laboratory Animals. Written consent was obtained prior to sample collection, and the Ethics Committee of the Shandong Provincial Hospital Affiliated to Shandong First Medical University approved this study. The experiment followed the Declaration of Helsinki (as revised in 2013).

Open Access Statement: This is an Open Access article distributed in accordance with the Creative Commons Attribution-NonCommercial-NoDerivs 4.0 International License (CC BY-NC-ND 4.0), which permits the noncommercial replication and distribution of the article with 


\section{Page 10 of 11}

the strict proviso that no changes or edits are made and the original work is properly cited (including links to both the formal publication through the relevant DOI and the license). See: https://creativecommons.org/licenses/by-nc-nd/4.0/.

\section{References}

1. Kim SU, Jeon MY, Lim TS. Diagnostic Performance of Serum Asialo- $\alpha 1$-acid Glycoprotein for Advanced Liver Fibrosis or Cirrhosis in Patients with Chronic Hepatitis B or Nonalcoholic Fatty Liver Disease. Korean J Gastroenterol 2019;74:341-8.

2. Afify SM, Tabll A, Nawara HM, et al. Five Fibrosis Biomarkers Together with Serum Ferritin Level to Diagnose Liver Fibrosis and Cirrhosis. Clin Lab 2018;64:1685-93.

3. Guo PF, Jin J, Sun X. Influence of IL10 gene polymorphisms on the severity of liver fibrosis and susceptibility to liver cirrhosis in HBV/HCV-infected patients. Infect Genet Evol 2015;30:89-95.

4. Basyte-Bacevice V, Skieceviciene J, Valantiene I, et al. SERPINA1 and HSD17B13 Gene Variants in Patients with Liver Fibrosis and Cirrhosis. J Gastrointestin Liver Dis 2019;28:297-302.

5. Basyte-Bacevice V, Skieceviciene J, Valantiene I, et al. TM6SF2 and MBOAT7 Gene Variants in Liver Fibrosis and Cirrhosis. Int J Mol Sci 2019;20:1277.

6. Fan YY, Ding W, Zhang C, et al. Obeticholic acid prevents carbon tetrachloride-induced liver fibrosis through interaction between farnesoid X receptor and Smad3. Int Immunopharmacol 2019;77:105911.

7. Gravito-Soares M, Gravito-Soares E, Gomes D, et al. Lysosomal Acid Lipase: Can it be a New Non-Invasive Serum Biomarker of Cryptogenic Liver Fibrosis and Cirrhosis? Ann Hepatol 2019;18:78-88.

8. Li Q, Yu B, Tian X, et al. Deep residual nets model for staging liver fibrosis on plain CT images. Int J Comput Assist Radiol Surg 2020;15:1399-406.

9. Kupcinskas J, Valantiene I, Varkalaite G, et al. PNPLA3 and RNF7 Gene Variants are Associated with the Risk of Developing Liver Fibrosis and Cirrhosis in an Eastern European Population. J Gastrointestin Liver Dis 2017;26:37-43.

10. $\mathrm{Li} \mathrm{H}$. Angiogenesis in the progression from liver fibrosis to cirrhosis and hepatocelluar carcinoma. Expert Rev Gastroenterol Hepatol 2021;15:217-33.

11. Zhang CY, Yuan WG, He P, et al. Liver fibrosis and hepatic stellate cells: Etiology, pathological hallmarks

\section{Zhang et al. The effects of miR-34a on the fibrosis of HSCs}

and therapeutic targets. World J Gastroenterol 2016;22:10512-22.

12. Higashi T, Friedman SL, Hoshida Y. Hepatic stellate cells as key target in liver fibrosis. Adv Drug Deliv Rev 2017;121:27-42.

13. Seo HY, Lee SH, Lee JH, et al. Src Inhibition Attenuates Liver Fibrosis by Preventing Hepatic Stellate Cell Activation and Decreasing Connetive Tissue Growth Factor. Cells 2020;9:558.

14. Cuiqiong W, Chao X, Xinling F, et al. Schisandrin B suppresses liver fibrosis in rats by targeting miR-101$5 p$ through the TGF- $\beta$ signaling pathway. Artif Cells Nanomed Biotechnol 2020;48:473-8.

15. Niu X, Nong $S$, Gong J, et al. Research on promoting liver fibrosis injury by the targeted regulation of miR-202 for HGF to activate HSC. Ir J Med Sci 2020;189:1295-304.

16. Zhao Y, Wang Z, Zhou J, et al. LncRNA Mical2/miR203a-3p sponge participates in epithelial-mesenchymal transition by targeting p66Shc in liver fibrosis. Toxicol Appl Pharmacol 2020;403:115125.

17. Li X, Zhang W, Xu K, et al. miR-34a promotes liver fibrosis in patients with chronic hepatitis via mediating Sirt1/p53 signaling pathway. Pathol Res Pract 2020;216:152876.

18. Lin HY, Wang FS, Yang YL, et al. MicroRNA29a Suppresses CD36 to Ameliorate High Fat DietInduced Steatohepatitis and Liver Fibrosis in Mice. Cells 2019;8:1298.

19. Lan T, Li C, Yang G, et al. Sphingosine kinase 1 promotes liver fibrosis by preventing miR-19b-3p-mediated inhibition of CCR2. Hepatology 2018;68:1070-86.

20. Verjans R, Peters T, Beaumont FJ, et al. MicroRNA-221/222 Family Counteracts Myocardial Fibrosis in Pressure Overload-Induced Heart Failure. Hypertension 2018;71:280-8.

21. Cui H, Ge J, Xie N, et al. miR-34a promotes fibrosis in aged lungs by inducing alveolarepithelial dysfunctions. Am J Physiol Lung Cell Mol Physiol 2017;312:L415-L424. Erratum in: Am J Physiol Lung Cell Mol Physiol 2018;314:L332.

22. Yu HJ, Liu W, Chang Z, et al. Probiotic BIFICO cocktail ameliorates Helicobacter pylori induced gastritis. World J Gastroenterol 2015;21:6561-71.

23. World Medical Association. World Medical Association Declaration of Helsinki: ethical principles for medical research involving human subjects. JAMA 2013;310:2191-4.

24. Ghavamzadeh A, Sotoudeh M, Hashemi Taheri AP, et al. Liver fibrosis alleviation after co-transplantation 
of hematopoietic stem cells with mesenchymal stem cells in patients with thalassemia major. Ann Hematol 2018;97:327-34.

25. King A, Houlihan DD, Kavanagh D, et al. Sphingosine1-Phosphate Prevents Egress of Hematopoietic Stem Cells From Liver to Reduce Fibrosis. Gastroenterology 2017;153:233-248.e16.

26. El-Akabawy G, El-Mehi A. Mobilization of endogenous bone marrow-derived stem cells in a thioacetamideinduced mouse model of liver fibrosis. Tissue Cell 2015;47:257-65.

27. Hu N, Guo C, Dai X, et al. Forsythiae Fructuse water extract attenuates liver fibrosis via TLR4/MyD88/NF- $\mathrm{BB}$ and TGF- $\beta /$ smads signaling pathways. J Ethnopharmacol 2020;262:113275.

28. Han X, Hong Y, Zhang K. TUG1 is involved in liver fibrosis and activation of HSCs by regulating miR-29b. Biochem Biophys Res Commun 2018;503:1394-400.

Cite this article as: Zhang $\mathrm{J}$, Wang $\mathrm{H}$, Yao L, Zhao $\mathrm{P}$, Wu X. MiR-34a promotes fibrosis of hepatic stellate cells via the TGF- $\beta$ pathway. Ann Transl Med 2021;9(20):1520. doi: 10.21037/atm-21-5005
29. Dávalos A, Goedeke L, Smibert P, et al. miR-33a/b contribute to the regulation of fatty acid metabolism and insulin signaling. Proc Natl Acad Sci U S A 2011;108:9232-7.

30. Hu J, Chen C, Liu Q, et al. The role of the miR-31/FIH1 pathway in TGF- $\beta$-induced liver fibrosis. Clin Sci (Lond) 2015;129:305-17.

31. Scorletti E, Afolabi PR, Miles EA, et al. Synbiotics Alter Fecal Microbiomes, But Not Liver Fat or Fibrosis, in a Randomized Trial of Patients With Nonalcoholic Fatty Liver Disease. Gastroenterology 2020;158:1597-1610.e7.

32. He S, Tang J, Diao N, et al. Parathyroid hormonerelated protein activates HSCs via hedgehog signalling during liver fibrosis development. Artif Cells Nanomed Biotechnol 2019;47:1984-94.

(English Language Editor: A. Kassem) 